\title{
Cache-Aided Millimeter Wave Ad-Hoc Networks
}

\author{
Satyanarayana Vuppala, Thang X. Vu, Sumit Gautam, Symeon Chatzinotas and Björn Ottersten \\ Interdisciplinary Centre for Security, Reliability and Trust, University of Luxembourgh, \\ 29, Avenue J.F Kennedy, L-1855 Luxembourg
}

\begin{abstract}
In this paper, we investigate the performance of cache enabled millimeter wave ( $\mathrm{mmWave}$ ) ad-hoc network, where randomly distributed nodes are supported by a cache memory. Specifically, we study the optimal caching placement at the desirable mmWave node using a network model that accounts for the uncertainties in node locations and blockages. We then characterize the average success probability of content delivery. As a desirable side effect, certain factors like the density of nodes and increased antenna gain, can significantly increase the cache hit ratio in mmWave networks. However, a trade-off between the cache hit probability and the average successful content delivery probability with respect to the density of nodes is presented.
\end{abstract}

Index Terms-Blockages, caching, interference, millimeterwave networks, Poison point processes

\section{INTRODUCTION}

The key objectives of future generation wireless communication systems include billions of connected devices, data rates in the range of Gbps, lower latencies, improved coverage and energy efficient operations. High user mobility is one of the expectations of such future communication networks where increase in the demand of content is primarily due to data applications like high quality video streaming and social networks. To meet the goals of this vision, cache-enabled network architectures are being investigated and projected as the possible solution to the inevitable data tsunami in coming years. The key idea in these systems is to avail the assistance from helper nodes instead of cellular infrastructures to successfully deliver the desired content to the end-user devices. Helper nodes are generally categorised as small base stations (SBSs) [1] and user mobile devices that are used for ad-hoc communications.

On the other hand, the existing cellular spectrum is approaching its performance limits thus giving birth to the growing interest in and exploration of supplementary resources to meet these demands [2]. As a result, millimeter wave (mmWave) frequencies are being investigated to serve as an alternative or provide assistance to the existing technologies. Propagation at higher frequencies is more hostile. Due to mmWave frequencies, the path losses are higher and more significant to blockages due to their smaller wavelengths. This was the reason why mmWave technology was discarded concerning its application in mobile communication. Nonetheless, high gain antenna arrays have been designed which can potentially overcome the losses. Tractable models for backhauled links and per user rates have been studied in [3], using stochastic geometry for their analysis.

The deployment of small cells is a key method by which wireless communication are expected to evolve as the next generation of networks [4]. MmWave transmission by nature can be deployed into ad-hoc mode due to its limited range of propagation. The work in [5] provides the coverage analysis of such mmWave ad-hoc networks whereas [6] provides coverage analysis in densification of mmWave cells. In addition, researchers are looking into how to maximize content delivery in networks to increase the users' quality of experience (QoE) [7]. In this vein, we consider the mmWave ad-hoc networks where individual nodes have storage units to cache popular contents. From the perspective of such mmWave networks, we demonstrate the effectiveness off-line caching with respect to blockages.

The idea of caching in mobile networks is triggering research interest as a promising approach to increase user satisfaction as well as reducing the cost of backhaul load. Caching has been used to maintain internet traffic over the last two decades and has been largely computer based [8]. This has triggered interest in the formulation of active caches for cellular networks. To this point, authors in [9] applied the content caching approaches to content delivery networks (CDN). Following this instinct, [10]-[13] show the role of proactive caching on the network edge to help reducing traffic congestion in the backhaul links. In particular, authors in [10] show the effectiveness of proactive caching in facilitating with reduction of congestion in the backhaul links wherein both spatial and social structures of networks has been considered to show that proactive caching aids users satisfaction.

The work in [11] shows that popularity based caching gives better results in terms of outage probability than uniform caching where the BSs cache content randomly irrespective of the popularity. Caching placement is a key factor that determines the success of a caching system, and researchers are looking into the optimal way of cache placement for various networks. In this direction, recently in [14], a caching placement that balances the reduction of network interference with channel selection diversity is proposed. All these previous works on caching have focused on transmission at frequencies of sub $6 \mathrm{GHz}$. Thus, in this work, we study the caching placement in mmWave networks where randomly located mmWave nodes store contents probabilistically and independently.

The main contributions are twofold, listed as follows

- In order to study the performance of mmWave ad-hoc cache enabled networks, we develop an analytical framework assuming a homogeneous Poisson Point Process (PPP) model of ad-hoc nodes with caching ability. From recent advances in the field and to the best of our knowledge, an absence of the cache-enabled mmWave ad-hoc networks may be noticed in the literature. 
- Next, we intend to maximize the cache hit probability, which is the probability that the typical receiver's request can be served by its neighbor nodes, by investigating the optimal caching placement in this mmWave ad-hoc network. Consequently, we characterize the average success probability of content delivery.

\section{System MODEL}

\section{A. Network model}

Consider a cache enabled wireless network where mmWave based transmitter nodes are modelled as a two dimensional homogeneous PPP $\Phi$ with intensity $\lambda$, each associated to a receiver. For simplicity, we refer to the receiver and the transmitter of the typical link as the typical receiver and the tagged transmitter respectively. Each node contains storage units that are used to cache popular files requested by other nodes. We refer to these units as the local caches. In addition, a central source containing a global cache consisting of all the files a user would require, is accessible to all the nodes in this network via wired backhaul links. This is to cater for the scenarios when the local caches do not contain the requested files. Using the Slivnyaks Theorem [15], it is found that for a given typical transmitter-receiver at the origin, the conditional distribution of the potential interferers (all transmitters excluding the tagged transmitter) is another homogeneous PPP with the same density $\lambda$.

Assumption 1: It is important to note that when a receiver requests a file, it is served either by the nearest node ${ }^{1}$ or best node which has the best average received power.

Blockage model: We leverage the concept of the blockage model from [16], and accordingly, consider a two state statistical model for each link. The link can be either LOS or nonline-of-sight (NLOS); LOS link occurs when there is a direct propagation path between the transmitter and the receiver, while NLOS occurs when the link is blocked and the receiver receives the signal through reflection from a blockage. Let the LOS link be of length $r$, then the probabilities of occurrence $p_{\mathrm{L}}($.$) and p_{\mathrm{N}}($.$) of LOS and NLOS states respectively can be$ given as a function of $r$ as

$$
p_{\mathrm{L}}(r)=e^{-\beta r}, \quad p_{\mathrm{N}}(r)=1-e^{-\beta r},
$$

where $\beta$ is the blockage density.

Beamforming model: Directional beamforming is implemented at both transmitters and receivers for cross communication in this system. The beam patterns are approximated to the sectorized gain patterns as in [5]. Let $\theta$ be the beamwidth of the main lobe. Then the antenna gain pattern for a transmit or receive node about an angle $\phi$ is given as

$$
G_{q}(\theta)=\left\{\begin{array}{ll}
G_{q}^{\mathrm{M}} & \text { if }|\phi| \leq \theta \\
G_{q}^{\mathrm{m}} & \text { if }|\phi| \geq \theta
\end{array}\right\}
$$

\footnotetext{
${ }^{1}$ We assume that the tagged transmitter associated to a typical receiver does not store the requested content. Therefore, the receiver seeks content from nearest or best available transmitter.
}

where $q \in \mathrm{T}, \mathrm{R}$ ( $\mathrm{T}$ denotes the transmitter, and $\mathrm{R}$ the receiver), $\phi \in[0,2 \pi)$ is the angle of boresight direction, $G_{q}^{\mathrm{M}}$ and $G_{q}^{\mathrm{m}}$ are the array gains of main and side lobes respectively.

The effective antenna gain for an interferer as seen by the typical receiver will depend on the directivity gains of the main (i.e., $G^{\mathrm{M}}$ ) and side (i.e., $G^{\mathrm{m}}$ ) lobes of the antenna beam pattern and is expressed as

$$
G_{i}=\left\{\begin{array}{ll}
G^{\mathrm{M}} G^{\mathrm{M}}, & \varsigma_{\mathrm{MM}}=\left(\frac{\theta}{2 \pi}\right)^{2} \\
G^{\mathrm{M}} G^{\mathrm{m}}, & \varsigma_{\mathrm{Mm}}=\frac{\theta(2 \pi-\theta)}{(2 \pi)^{2}} \\
G^{\mathrm{m}} G^{\mathrm{M}}, & \varsigma_{\mathrm{mM}}=\frac{\theta(2 \pi-\theta)}{(2 \pi)^{2}} \\
G^{\mathrm{m}} G^{\mathrm{m}}, & \varsigma_{\mathrm{mm}}=\left(\frac{2 \pi-\theta}{2 \pi}\right)^{2}
\end{array}\right\},
$$

where $\varsigma_{l k}$, with $l, k \in\{\mathrm{M}, \mathrm{m}\}$ denotes the probability that the antenna gain $G^{l} G^{k}$ is seen by the receiver. Further, we assume that the transmitter of every link is spatially aligned with its intended receiver as considered in [16].

Channel model: For analytical tractability, we consider Nakagami fading model as it is commonly used in the literature [5], [16]. Hence, the channel power is distributed according to gamma random variable with $\nu$ is the Nakagami fading parameter.

For simplicity, we represent the size of a cache by the size of its library, that is the total number of contents in the library is $L$. Thus, the set of contents is denoted as $\mathcal{L}=\{1,2,3, \ldots, L\}$. All files are assumed to be equal length and unit size. The more popular contents in the library with $L$ contents are requested with a higher likelihood.

Caching model: The requests from receivers to the transmitters are assumed to be independent of each other. Let $f_{j}$ be the probability that a user requests the $j$-th file in the library. This probability is defined according to Zipf distribution [17] as

$$
f_{j}=\frac{j^{-\Upsilon}}{\sum_{i=1}^{L} i^{-\Upsilon}}, \quad 1 \leq j \leq L,
$$

where $\Upsilon$ is the Zipf exponent which controls the popularity of files with large values of $\Upsilon$ indicating a higher content reuse.

We assume random caching placement where the ad-hoc node stores or caches the content $j$ with probability $q_{j}$ for all requested files, i.e., $\forall j \in \mathcal{L}$. The cache size is denoted with $\mathcal{M}$. Consider $\mathbf{q}=\left[q_{1}, \cdots, q_{L}\right]$ as the caching probabilities of the file $i \in[1, L]$, and constraint is $\sum_{j=1}^{L} q_{j} \leq \mathcal{M}$. Readers are encouraged to refer [18] for more details on random content placement. Under the assumption of caching request or overhearing, each content is cached at transmitter node in probabilistic way. Note that each node independently stores the content according to the caching distribution. The transmitter nodes storing the content $j$ can be modeled as an independent PPP with intensity $q_{j} \lambda$, where the locations of all transmitter nodes storing the content $j$ are denoted by $\Phi_{j}$.

Consider an event when the receiver (user) requested file is not cached in given tagged transmitter. With such an assumption, we define two types of caching hit events as follows

- Case 1: nearest node, when the requested file is cached at nearest mmWave LOS transmitter node. 
- Case 2: best node, when the requested file is not found in nearby mmWave transmitter nodes but in one of its closest LOS transmitter nodes with the least average received path loss.

The proposed cahe hit events are distinct from each other since the best node scenario depends on the channel conditions while nearest node scenario relies only on distance.

\section{B. Performance metrics}

The metric that we consider in this paper, is the average success probability which is defined forthwith.

Average successful content delivery probability: This is defined as the successful response to the typical receiver's file request when the requested file is successfully downloaded. The successful content delivery probability of a requested file defined as the probability that a receiver succeeds in decoding the received content from its associated transmitter. Thus when the typical receiver requests the $j$-th file from its serving node, given the received SINR, and the SINR target $\Theta_{j}$, the successful content delivery probability is expressed as

$$
\mathcal{P}_{\text {suc }}^{j}\left(\Theta_{j}\right)=p\left[\frac{P_{m} G_{l}\left|h_{l}\right|^{2} r_{l}-\alpha_{m}}{\sigma^{2}+I_{j}} \geq \Theta_{j}\right],
$$

where $G_{l}$ is the antenna array gain function, $h_{l}$ is the fading gain at the receiver of interest, $r_{l}$ is the link length, $\sigma^{2}$ is the noise power, and $I_{j}$ is the interference received by the typical receiver.

Let $\Phi_{j}^{c}\left(\triangleq \Phi \backslash \Phi_{j}\right)$ to be another set of interfering locations with density $\left(1-q_{j}\right) \lambda$, i.e., interference from all other ad-hoc nodes which do not cache content $j$ in their cache memory. Therefore, the received SINR for the typical receiver can now be defined as

$$
\bar{\zeta}_{j} \triangleq \frac{P_{m} G_{l}\left|h_{l}\right|^{2} r_{l}{ }^{-\alpha_{m}}}{\sigma^{2}+I_{j}}
$$

where

$$
I_{j}=\underbrace{\sum_{i \in \Phi_{j}} P_{m} G_{i}\left|h_{i}\right|^{2} r_{i}^{-\alpha_{m}}}_{I_{j-\text { In }}}+\underbrace{\sum_{i \in \Phi_{j}^{c}} P_{m} G_{i}\left|h_{i}\right|^{2} r_{i}^{-\alpha_{m}}}_{I_{j-\text { Non }}},
$$

and $h_{i}$ denotes each interference fading gain, $r_{i}$ is the distance from the interferer $i$ to the typical receiver.

By averaging over all file requests, the average successful content delivery probability is given as

$$
\mathcal{P}_{\text {suc }}(\Theta)=\sum_{j=1}^{L} f_{j} \cdot \mathcal{P}_{\text {suc }}^{j}\left(\Theta_{j}\right),
$$

where $f_{j}$ is the probability of requesting the $j$ th file.

\section{Cache Hit Probability \& Successful Content DELIVERY ANALYSIS}

In this section, we characterize the cache hit probabilities required for a cache aided transmission in mmWave networks. To model the caching dynamics of a given node, we use the commonly used path loss distributions which helps us to derive the cache hit probability. As discussed in the earlier section, the caching hit probability is characterized in two cases that are presented in the further subsections.

\section{A. Cache hit characterization}

1) Nearest node: Consider $\mathcal{P}_{h}^{n}$ as the caching hit probability in the case of nearest node. Due to probabilistic caching assumption, the probability to find a cached file in given area is strongly dependent on blockages and the coverage area of ad-hoc cell. Hence we present the nearest node distance distribution with blockages in the following lemma.

Lemma 1. The nearest distance distribution for the case of LOS mmWave networks is given as

$$
F\left(r ; q_{j}\right)=1-\exp \left(-\frac{2 \pi q_{j} \lambda\left(1-e^{-\beta r}(\beta r+1)\right)}{\beta^{2}}\right) .
$$

Proof. Due to space constraints, the proof of this lemma is omitted.

Therefore, when a user requests a file $j$ with a probability $f_{j}$ and its caching probability $q_{j}$, the probability of finding that file in the nearest transmitter node is given as

$$
\mathcal{P}_{h, j}^{n}=1-\exp \left(-\frac{2 \pi q_{j} \lambda\left(1-e^{-\beta r}(\beta r+1)\right)}{\beta^{2}}\right) .
$$

Averaging over all the files, the cache hit probability is given as

$$
\mathcal{P}_{h}^{n}=\sum_{i=1}^{L} f_{j}\left(1-q_{j}\right) F\left(r ; q_{j}\right)
$$

Hence, we study the optimal caching probabilities for cache hit maximization. The optimization problem for maximizing the cache hit probability is given as

$$
\begin{aligned}
\mathbf{P 1}: \max _{\mathbf{q}} & \mathcal{P}_{h}^{n} \\
\text { s.t. } & 0 \leq q_{j} \leq 1 \text { for } j=1, \cdots, L \\
& \sum_{j=1}^{L} q_{j} \leq \mathcal{M}
\end{aligned}
$$

We derive the optimal solution of P1, i.e., the optimal caching probabilities, in closed-form. The second order derivative of $\mathrm{P} 1$ is strictly negative, thus $\mathcal{P}_{h}^{n}$ is a concave function for each $j$. Since weighted sum of convex functions is also convex function, P1 is a constrained convex optimization problem thus a unique solution exists. Hence the Lagrangian function of P1 is given as

$$
\begin{array}{r}
\mathcal{L}\left(q_{j}, \omega\right)=-1+\sum_{j=1}^{L} f_{j}\left(1-q_{j}\right) e^{\frac{2 \pi q_{j} \lambda\left(1-e^{-\beta r}(\beta r+1)\right)}{\beta^{2}}} \\
+\omega\left(\sum_{i=1}^{L} q_{j}-\mathcal{M}\right),
\end{array}
$$

where $\omega$ is the Lagrangian multiplier. This constrained optimization problem can be solved by applying the KarushKuhn-Tucker (KKT) conditions. Therefore, after differentiating $\mathcal{L}\left(q_{j}, \omega\right)$ with respect to $q_{j}$, we can obtain all the necessary 
KKT conditions. The optimal caching probabilities can be given as

$$
q_{j}(\omega)=-\frac{\mathcal{W}\left(\frac{\omega}{q_{j}} \Xi\right)}{\Xi}+\frac{1}{\Xi}+1
$$

where $\Xi=\frac{2 \pi \lambda\left(-1+e^{-\beta r}(\beta r+1)\right)}{\beta^{2}}$ and $\mathcal{W}$ denotes the Lambert W function [19]. By applying the KKT condition again, we have

$$
q_{j}^{*}(\omega)=\min \left\{\left[q_{j}\left(\omega^{*}\right)\right]^{+}, 1\right\},
$$

where $[x]^{+}=\max \{x, 0\}$ and $\omega^{*}$ can be obtained by the Bisection method [14, Algorithm 1].

2) Best node: Taking into account the path loss during the communication between a given receiver and mmWave transmitter link, the minimum power received at the receiver is

$$
\xi_{l}=P_{m} G_{i} r^{\alpha_{\mathrm{L}}} .
$$

Before advancing further, it is noteworthy that (16) is important in the context of cache hit probability characterization. Also, the actual received signal power at the receiver may be reflected by the uncertainties like path loss, shadowing, and other fading factors, which causes a phenomenon commonly known as the ping-pong [20] effect. This outcome is avoided by using the long-term averaged power [20], which is determined by taking the mean of the received signal over a given period of time. Therefore, the least pathloss distribution in a mmWave network is not the same as for the case of a cellular network.

Lemma 2. The least path loss distribution in a LOS mmWave network can be given as

$$
\begin{aligned}
F_{\xi_{l}}\left(r ; q_{j}\right)= & 1-\prod_{l, k \in\{\mathrm{M}, \mathrm{m}\}} \exp \left(-\frac{2 \pi p_{l k} q_{j} \lambda}{\beta^{2}}\right. \\
& \left.\left.\times\left(1-e^{-\beta\left(r P_{m} G_{i}^{l k}\right)^{\frac{1}{\alpha_{L}}}}\left(1+\beta\left(r P_{m} G_{i}^{l k}\right)^{\frac{1}{\alpha_{L}}}\right)\right)\right)\right) .
\end{aligned}
$$

Proof. The proof of this lemma can be obtained from [21].

Averaging over all the files, the cache hit probability is given as

$$
\mathcal{P}_{h}^{b}=\sum_{i=1}^{L} f_{i}\left(1-q_{j}\right) F_{\xi_{l}}\left(r ; q_{j}\right)
$$

Hence, we study the optimal caching probabilities for cache hit maximization. The optimization problem for maximizing the cache hit probability is give as

$$
\begin{aligned}
\mathbf{P 2}: \max _{\mathbf{q}} & \mathcal{P}_{h}^{b} \\
\text { s.t. } & 0 \leq q_{j} \leq 1 \text { for } j=1, \cdots, L \\
& \sum_{j=1}^{L} q_{j} \leq \mathcal{M}
\end{aligned}
$$

The optimal solution of P2 can be solved by following similar approach in characterizing P1. Hence, for brevity, we have omitted the corresponding proof.

\section{B. Average Successful Content Delivery Probability}

In the following proposition, we present the success probability at the typical receiver from a given mmWave transmitter for a predefined SINR target $\Theta_{j}$.

Proposition 1. The success probability of content delivery at the typical receiver from a given mmWave LOS transmitter node is given as

$\mathcal{P}_{\text {suc }}^{j}\left(\Theta_{j}\right)=\sum_{k=0}^{\nu}\left(\begin{array}{l}\nu \\ k\end{array}\right)(-1)^{k} \int_{y>0} e^{\frac{-A k \Theta_{j} y^{\alpha} \sigma^{2}}{P_{m} G_{l}}}$
$\times \prod_{t \in \mathrm{L}, \mathrm{N}} \mathbb{E}_{\mathcal{I}_{j-\mathrm{In}}^{t}}\left[e^{\frac{-A k y^{\alpha} \Theta_{j} \mathcal{I}_{j-\mathrm{In}}^{t}}{P_{m} G_{l}}}\right] \mathbb{E}_{\mathcal{I}_{j-\mathrm{Non}}^{t}}\left[e^{\frac{-A k y^{\alpha} \Theta_{j} \mathcal{I}_{j-\mathrm{Non}}^{t}}{P_{m} G_{l}}}\right] f(y) d y$,

where $\nu$ is a parameter from the tight upper bound of Gamma distribution given as $\mathcal{P}\left[\left|h_{l}\right|^{2}<\gamma<\left(1-e^{-A \gamma}\right)^{\nu}\right]$ with $A=$ $\nu(\nu !)^{\frac{-1}{\nu}}, f($.$) can be obtained by taking derivative of (9) and$ $\mathbb{E}_{\mathcal{I}_{j-\text { In }}^{t}}[$.$] follows from (23).$

\section{Proof.}

The average success probability conditioned on the least path loss from the best node to the typical receiver is defined as

$$
\mathcal{P}_{\text {suc }}^{j}\left(\Theta_{j}\right)=\int_{y>0} \mathcal{P}\left[\frac{P_{m} G_{l}\left|h_{l}\right|^{2} y^{-\alpha}}{\sigma^{2}+I_{j}}>\Theta_{j}\right] f(y) \mathrm{dy} .
$$

Given that the small scale fading $\left|h_{l}\right|$, is Nakagami, and employs the upper bound of gamma distribution with parameter $\nu$ such that: $\mathcal{P}\left[\left|h_{l}\right|^{2}<\gamma<\left(1-e^{-A \gamma}\right)^{\nu}\right]$ with $A=\nu(\nu !)^{\frac{-1}{\nu}}$, therefore, the average success probability is expressed as:

$$
\begin{gathered}
\mathcal{P}\left[\frac{P_{m} G_{l}\left|h_{l}\right|^{2} y}{\sigma^{2}+I_{j}}>\Theta_{j}\right]=\sum_{k=0}^{\nu}\left(\begin{array}{l}
\nu \\
k
\end{array}\right)(-1)^{k} e^{\frac{-A k y^{\alpha} \Theta_{j} \sigma^{2}}{P_{m} G_{l}}} \\
\times \prod_{t \in \mathrm{L}, \mathrm{N}} \mathbb{E}_{\mathcal{I}_{j-\text { In }}^{t}}\left[e^{\frac{-A k y^{\alpha} \Theta_{j} \mathcal{I}_{j}^{t}}{P_{m} G_{l}}}\right] \mathbb{E}_{\mathcal{I}_{j-\mathrm{Non}}^{t}}\left[e^{\frac{-A k y^{\alpha} \Theta_{j} \mathcal{I}_{j}^{t}}{P_{m} G_{l}}}\right],
\end{gathered}
$$

which follows from applying binomial expansion, and due to the fact that interference links can be LOS or NLOS such that $\mathcal{I}_{j}=\mathcal{I}_{j-\text { In }}^{\mathrm{L}}+\mathcal{I}_{j-\text { Non }}^{\mathrm{N}}$.

Applying the probability generating functional of PPP (PGFL) [15], we obtain:

$$
\begin{aligned}
\mathbb{E}_{\mathcal{I}_{j-\mathrm{In}}^{\mathrm{L}}} & {\left[\exp \left(\frac{\left.\left.-A k y^{\alpha} \Theta_{j} \mathcal{I}_{j-\mathrm{In}}^{\mathrm{L}}\right)\right]=\prod_{l, k \in\{\mathrm{M}, \mathrm{m}\}} \exp (-2 \pi}{P_{m} G_{l}}\right)\right.} \\
& \left.\times \varsigma_{l k} q_{j} \lambda \int_{0}^{\infty}\left(1-\frac{1}{\left(1+\frac{A k G_{i}^{l k} \Theta_{j} y^{\alpha}}{P_{m} G_{l} r^{\alpha} \mathrm{L}}\right)^{\nu}} p_{\mathrm{L}}(r) \mathrm{dr}\right)\right) .
\end{aligned}
$$

The expectation of NLOS interfering link can be obtained similarly. Therefore, the proof concludes.

Consequently, the content average success probability maximized with $q_{j}^{*}(\omega)$ is given as

$$
\mathcal{P}_{\text {suc }}^{*}(\Theta)=\sum_{j=1}^{L} f_{j} \cdot \mathcal{P}_{\text {suc }}^{j}\left(\Theta_{j} ; q_{j}^{*}(\omega)\right) \text {. }
$$




\section{NUMERICAL ANALYSIS}

In general, the computations are performed through Monte Carlo simulations, which are then used to validate the analytical results. Unless stated otherwise, most of the values of the parameters used are inspired from literature mentioned in the references [5], [16]. A few of the parameters and their corresponding values are given as $L=5, \mathcal{M}=3, \gamma=1.4$, $\beta=0.0143, \lambda=0.0005, G^{M}=10 \mathrm{~dB}, G^{m}=0 \mathrm{~dB}$, and $P_{m}=1$ Watt, $\alpha_{\mathrm{L}}=2.1$ and $\alpha_{\mathrm{N}}=3.5$. All other parameters and values will be explicitly mentioned wherever used.

First we compare the cache hit probability with different node selections and for various blockage densities. These results validate lemma 1 and 2. Fig. 1 depicts the analytical results for the variation in the cache hit probability with increasing density of ad-hoc nodes $(\lambda)$. Clear distinction between the random file caching, and optimal file caching is presented for the nearest and best selection criterion. In the nearest selection, the ad-hoc node closest to the typical receiver is selected while in the best selection, an ad-hoc node is chosen based on the finest channel conditions. It is seen that the cache-hit probability increases with the increase in number of ad-hoc nodes, which is obvious as intuitively the probability of storing the files becomes high with increase in the ad-hoc nodes.

Fig. 2 illustrates the transition in the values of cache hit probability with increasing density of ad-hoc nodes $(\lambda)$ for different values of blockage density $(\beta)$. Same as in Fig. 1, a divergence analysis is illustrated for best and nearest adhoc node selections but implementing the random, no, and directional beamforming methods. It is seen that the cache hit probability decreases with increasing values of $\beta$. However as expected, the results of directional beamforming method with proper beam alignment between the transmitter and receiver outperforms both the no beamforming method with omnidirectional radiation and random beamforming method with non-alignment of beams. Additionally, it is observed that the random beamforming method performs better than the no beamforming method for decreasing $\beta$. It is also seen that the blockages have an adverse effect on cache hit probability.

Fig. 3 presents the plot of the cache hit probability against the order of files for various selections of caching size $(M)$ and Zipf coefficient $(\gamma)$. It is found that the cache hit probability increases with the increasing $M$ and $\gamma$ values. However, the cache hit probability declines considerably according to the parameter value selections of $M$ and $\gamma$ as the order of files within the ad-hoc node increases.

After establishing the effect of cache hit probability in the previous figures, we now look into the average successful delivery probability. Fig. 4 highlights the average success probability of content delivery versus SINR (in $\mathrm{dB}$ ) for different values of $G_{l}$ at the receiver. It is found that the average success probability of content delivery increases with increasing $G_{l}$ at the receiver. On the contrary and as expected, it is observed that the average success probability of content delivery decreases considerably with increasing SINR threshold values.

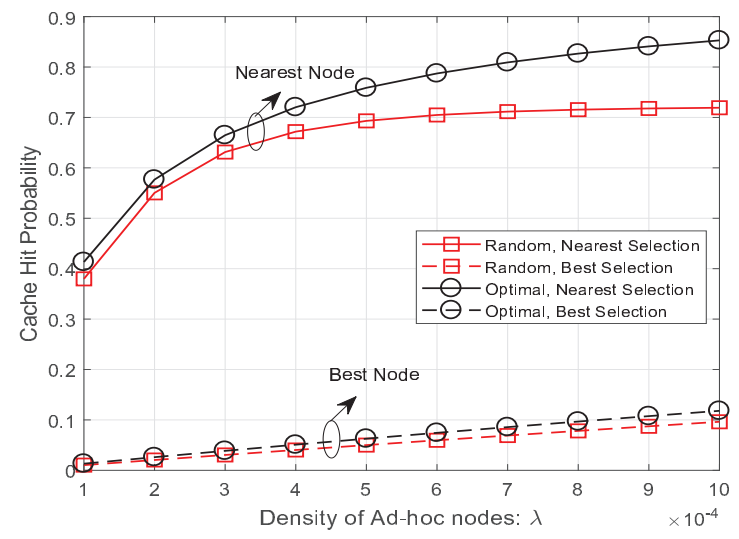

Fig. 1. The Cache Hit Probability versus $(\lambda)$ for different criterion with random and optimal selections of file caching.

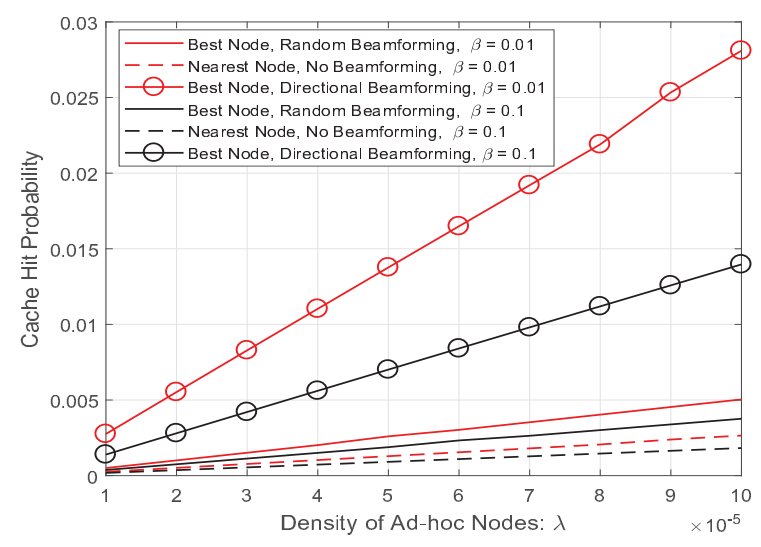

Fig. 2. The Cache Hit Probability versus $\lambda$ considering random, none, and directional beamforming methods.

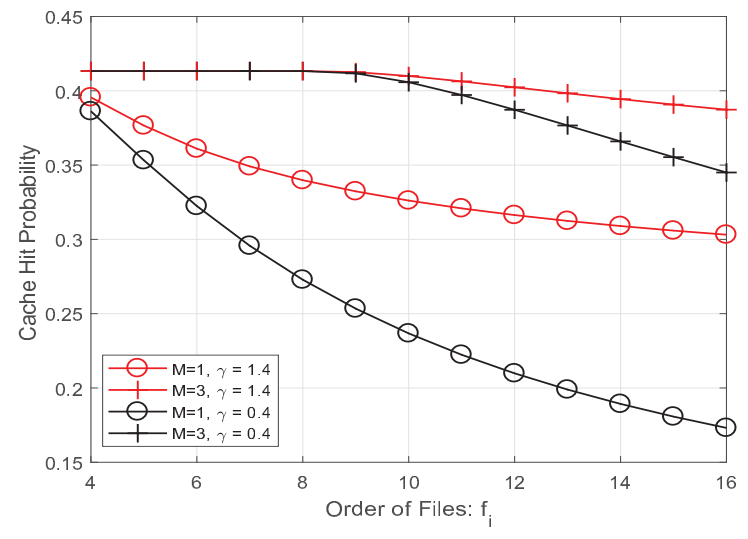

Fig. 3. The Cache Hit Probability versus request probability $\left(f_{i}\right)$.

Fig. 5 plots the average success probability of content delivery as a function node density. It is clear from the figure that the LOS path loss exponent has minor effect on the the 


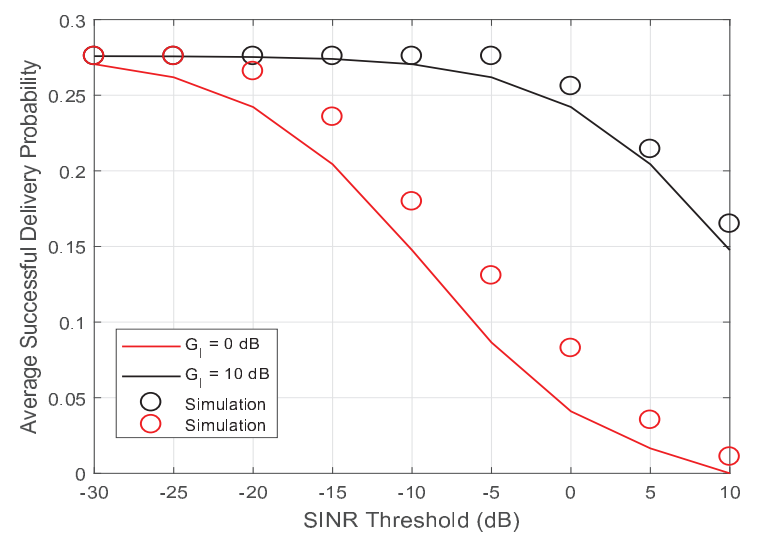

Fig. 4. Average successful delivery probability versus SINR for various $G_{l}$.

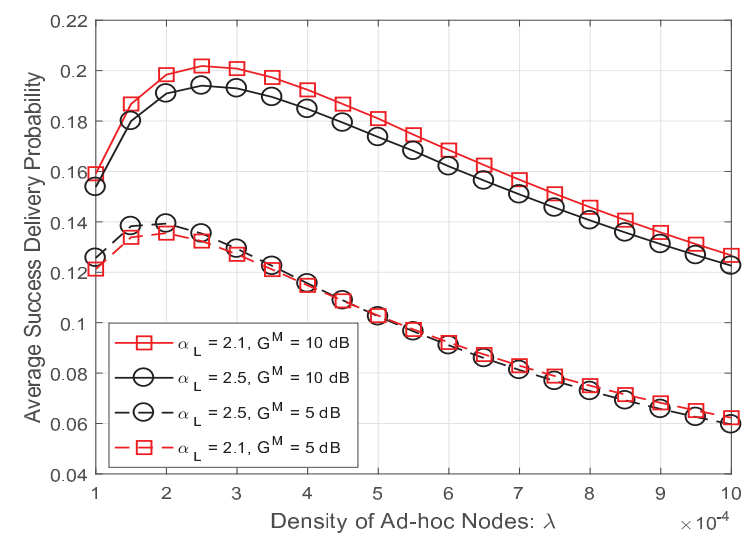

Fig. 5. Average success probability of content delivery versus $\lambda$.

average success probability. It can be explained from the fact that the mmWave LOS channel is less likely change due to channel conditions. It is worthy to mention that there is an optimal value of the average success probability of content delivery as indicated by the shape of the curves in Fig. 5 with the implication that increasing the ad-hoc node density has a diminishing returns effect. Analytical determination of this optimal point can be explored in future works.

\section{CONCLUSION}

The potential benefits of deploying cache enabled ad-hoc nodes in outdoor mmWave networks were investigated. From our analysis, it is clear that the cache hit probability depends on the blockage density and network conditions such as path loss exponent, antenna gain, and density of the ad-hoc nodes. Accordingly, the average successful content delivery probability for the ad-hoc nodes was studied. Since interference increases with the number of nodes, a trade-off can be observed in the cache hit probability and the content delivery probability with respect to the density of nodes.

\section{ACKNOWLEDGEMENT}

This work has received funding from the European Research Council (ERC) under the European Union's Horizon H2020 research and innovation programme (grant agreement No 742648).

\section{REFERENCES}

[1] K. Shanmugam, N. Golrezaei, A. G. Dimakis, A. F. Molisch, and G. Caire, "Femto caching: Wireless content delivery through distributed caching helpers," vol. 59, pp. 8402-8413, 2013.

[2] D. Tse and P. Viswanath, Fundamentals of wireless communication. Cambridge university press, 2005.

[3] S. Singh, M. N. Kulkarni, A. Ghosh, and J. G. Andrews, "Tractable model for rate in self-backhauled millimeter wave cellular networks," IEEE J. Select. Areas Commun., vol. 33, no. 10, pp. 2196-2211, 2015.

[4] N. Bhushan, J. Li, D. Malladi, R. Gilmore, D. Brenner, A. Damnjanovic, R. T. Sukhavasi, C. Patel, and S. Geirhofer, "Network densification: the dominant theme for wireless evolution into 5G," IEEE Commun. Mag., vol. 52, no. 2, pp. 82-89, 2014.

[5] A. Thornburg, T. Bai, and R. W. Heath, "Performance analysis of mmWave ad hoc networks," IEEE Trans. Signal Process., vol. 64, no. 15, pp. 4065 - 4079, Apr. 2016.

[6] E. Turgut and M. C. Gursoy, "Coverage in heterogeneous downlink millimeter wave cellular networks," to appear in IEEE Trans. on Commun., vol. pp, 2017.

[7] J. Song, H. Song, and W. Choi, "Optimal caching placement of caching system with helpers," in IEEE International Conference on Communications (ICC), June 2015, pp. 1825-1830.

[8] G. Ananthanarayanan, A. Ghodsi, A. Wang, D. Borthakur, S. Kandula, S. Shenker, and I. Stoica, "Pacman: coordinated memory caching for parallel jobs," in Proceedings of the 9th USENIX conference on Networked Systems Design and Implementation. USENIX Association, 2012, pp. 20-20.

[9] G. Paschos, E. Bastug, I. Land, G. Caire, and M. Debbah, "Wireless caching: Technical misconceptions and business barriers," IEEE Commun. Magazine, vol. 54, no. 8, pp. 16-22, 2016.

[10] E. Bastug, M. Bennis, and M. Debbah, "Living on the edge: The role of proactive caching in 5G wireless networks," IEEE Commun. Mag., vol. 52, no. 8, pp. 82-89, 2014.

[11] S. T. ul Hassan, M. Bennis, P. H. J. Nardelli, and M. Latva-aho, "Caching in wireless small cell networks: A storage-bandwidth tradeoff," IEEE Communications Letters, vol. 20, no. 6, pp. 1175-1178, June 2016.

[12] O. Semiari, W. Saad, M. Bennis, and B. Maham, "Caching meets millimeter wave communications for enhanced mobility management in 5G networks," 2017. [Online]. Available: arXiv:1701.05125

[13] T. X. Vu, S. Chatzinotas, B. Ottersten, and T. Q. Duong, "Energy minimization for cache-assisted content delivery networks with wireless backhaul," IEEE Wireless Commun. Lett., vol. pp, no. pp, Nov. 2017.

[14] S. H. Chae and W. Choi, "Caching placement in stochastic wireless caching helper networks: Channel selection diversity via caching," IEEE Transactions on Wireless Communications, vol. 15, no. 10, pp. 66266637, Oct 2016.

[15] M. Haenggi, Stochastic geometry for wireless networks. Cambridge University Press, 2012.

[16] T. Bai and R. W. Heath, "Coverage and rate analysis for millimeter-wave cellular networks," IEEE Trans. Wireless Commun., vol. 14, no. 2, pp. 1100-1114, Feb. 2015.

[17] M. Penrose, Random geometric graphs. Oxford University Press, 2003, no. 5 .

[18] B. Blaszczyszyn and A. Giovanidis, "Optimal geographic caching in cellular networks," in 2015 IEEE International Conference on Communications (ICC), June 2015, pp. 3358-3363.

[19] R. M. Corless, G. H. Gonnet, D. E. G. Hare, D. J. Jeffrey, and D. E. Knuth, "On the lambert w function," Advances in Conputational Mathematics, vol. 5, no. 1, pp. 329-359, 1996.

[20] H.-S. Jo, Y. J. Sang, P. Xia, and J. G. Andrews, "Heterogeneous cellular networks with flexible cell association: A comprehensive downlink SINR analysis," IEEE Trans. Wireless Commun., vol. 11, no. 10, pp. 34843495, Oct. 2012.

[21] D. Maamari, N. Devroye, and D. Tuninetti, "Coverage in mmWave cellular networks with base station cooperation," IEEE Trans. Wireless Comm., vol. 15, pp. 2981-2994, Jan. 2016. 